\title{
Characterization of oxylipins and dioxygenase genes in the asexual fungus Aspergillus niger
}

\author{
Mayken W Wadman*1,2, Ronald P de Vries ${ }^{2}$, Stefanie IC Kalkhove ${ }^{2}$, \\ Gerrit A Veldink ${ }^{1}$ and Johannes FG Vliegenthart ${ }^{1}$
}

\author{
Address: ${ }^{1}$ Bioorganic Chemistry, Utrecht University, $3584 \mathrm{CH}$, Utrecht, the Netherlands and ${ }^{2}$ Microbiology, Utrecht University, $3584 \mathrm{CH}, \mathrm{Utrecht}$ \\ the Netherlands \\ Email: Mayken WWadman*-m.w.wadman@uu.nl; Ronald P de Vries - r.p.devries@uu.nl; Stefanie IC Kalkhove - s.i.c.kalkhove@uu.nl; \\ Gerrit A Veldink - g.a.veldink@uu.nl; Johannes FG Vliegenthart - j.f.g.vliegenthart@uu.nl \\ * Corresponding author
}

Published: 23 March 2009

BMC Microbiology 2009, 9:59 doi:10.1/86/147|-2180-9-59

This article is available from: http://www.biomedcentral.com/I47I-2/80/9/59

(c) 2009 Wadman et al; licensee BioMed Central Ltd.

This is an Open Access article distributed under the terms of the Creative Commons Attribution License (http://creativecommons.org/licenses/by/2.0), which permits unrestricted use, distribution, and reproduction in any medium, provided the original work is properly cited.
Received: 17 September 2008

Accepted: 23 March 2009

\begin{abstract}
Background: Aspergillus niger is an ascomycetous fungus that is known to reproduce through asexual spores, only. Interestingly, recent genome analysis of $A$. niger has revealed the presence of a full complement of functional genes related to sexual reproduction [I]. An example of such genes are the dioxygenase genes which in Aspergillus nidulans, have been shown to be connected to oxylipin production and regulation of both sexual and asexual sporulation [2-4]. Nevertheless, the presence of sex related genes alone does not confirm sexual sporulation in A. niger.
\end{abstract}

Results: The current study shows experimentally that $A$. niger produces the oxylipins $8,1 \mathrm{I}$ dihydroxy octadecadienoic acid (8,II-diHOD), 5,8-dihydroxy octadecadienoic acid (5,8-diHOD), lactonized 5,8-diHOD, 8-hydroxy octadecadienoic acid (8-HOD), 10-hydroxy octadecadienoic acid (I0-HOD), small amounts of 8-hydroxy octadecamonoenoic acid (8-HOM), 9-hydroxy octadecadienoic acid (9-HOD) and 13-hydroxy octadecadienoic acid (I3-HOD). Importantly, this study shows that the $A$. niger genome contains three putative dioxygenase genes, $p p o A, p p o C$ and ppoD. Expression analysis confirmed that all three genes are indeed expressed under the conditions tested.

Conclusion: A. niger produces the same oxylipins and has similar dioxygenase genes as A. nidulans. Their presence could point towards the existence of sexual reproduction in $A$. niger or a broader role for the gene products in physiology, than just sexual development.

\section{Background}

The fungal kingdom comprises a large group of organisms (estimated to consist of over 1.5 million species) with only $5 \%$ identified thus far. Fungal species can survive in virtually all biotopes on earth, as they have been identified in water and soil, and on plants and animals. Part of their success comes from the ability to use different reproductive strategies, which provide increased flexibility for diverse environmental requirements. Fungal species can produce sexual cells and/or asexual cells in distinct reproductive structures. Some fungi are able to reproduce both sexually and asexually depending on the circumstances, while others display one mode of reproduction, only. Sexual reproduction and recombination allows the repair of naturally occurring mutations and results in new genotypes and phenotypes that allow for natural selection [5]. On the other 
hand, asexual reproduction provides the ability to disperse numerous genetically identical mitospores, without the metabolic costs of sexual reproduction [5].

Aspergillus niger is an ascomycetous fungus that is considered to reproduce through asexual spores, only. Since $A$. niger is used as a host for the production of homologous and heterologous proteins and commercially important compounds (such as citric acid), the potential presence of a sexual cycle is highly significant for strain improvement. Recent analysis of the A. niger genome has revealed the presence of a full complement of genes related to sexual reproduction [1]. It was therefore suggested that there could be a latent sexual potential in A. niger. A similar observation applies to Aspergillus fumigatus and Aspergillus oryzae, both only known to reproduce asexually, so far. Comparison of the two genomes to the genome of Aspergillus nidulans (please note that the holomorph is correctly named Emericella nidulans, but is hereafter mentioned as A. nidulans), which has a known sexual cycle, suggests that both $A$. fumigatus and A. oryzae may be capable of sexual reproduction [6]. It has yet to be determined whether genes related to sexual reproduction in supposedly asexual fungi are functional.

Dioxygenase genes with homology to mammalian prostaglandin synthase (PGS) have been connected to the formation of oxylipins in A. nidulans. Dioxygenase genes and oxylipins are linked to reproduction as they regulate the balance between sexual and asexual sporulation [2-4]. The goal of this study was to investigate whether or not oxylipins and dioxygenase genes related to sexual reproduction are also present in the asexual fungus $A$. niger.

\section{Results \\ RP-HPLC analysis}

A crude extract of $A$. niger $\mathrm{N} 402$ biomass was incubated with 18:2 and the reaction mixture was extracted with SPE and analyzed on RP-HPLC. A typical HPLC chromatogram is shown in Fig. 1. Incubation with 18:2 resulted in the appearance of three large peaks in the HPLC chromatogram and a smaller one. Similar results were obtained for A. niger UU-A049.1, A. niger $\triangle p p o A$ (UU-A050.3), A. niger $\triangle p p o D$ (UU-A051.26) and A. nidulans WG096 (data not shown). For each strain, fatty acid reaction products were fractionated on HPLC and after derivatization further investigated with GC/MS. Structures of oxygenated fatty acids were deduced from the spectra of the TMS ethers of methyl ester derivatives.

\section{GCIMS analysis of dihydroxy fatty acids (RP-HPLC peak I, peak 2 and peak $2 *$ )}

Hydrogenated dihydroxy fatty acids as TMS ethers of methyl ester derivatives from RP-HPLC peak 1 (Fig. 1) were separated on GC and one dominant peak was present in the chromatogram. The mass spectrum was

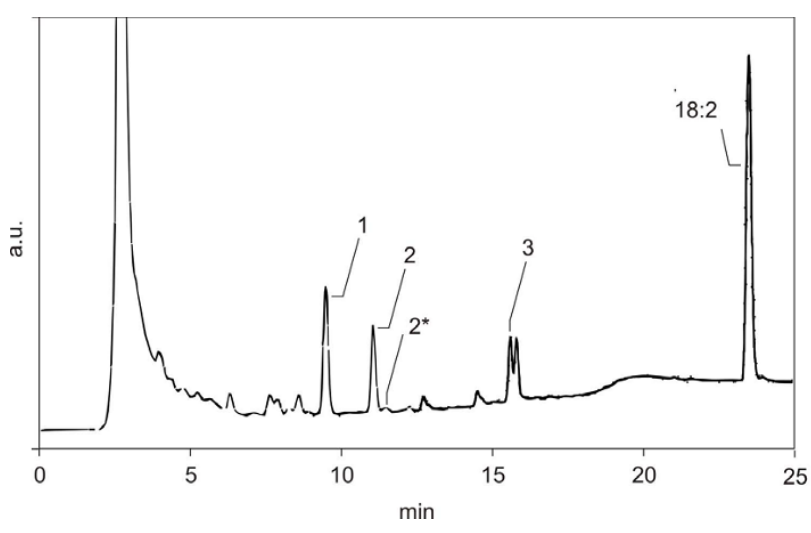

Figure I

RP-HPLC chromatogram $(\lambda=200 \mathrm{~nm})$ of the reaction of a crude extract of $A$. niger $\mathbf{N 4 0 2}$ biomass with I 8:2. Indicated are peak I (9.2 min; 8, I I-diHOD), peak 2 (I0,8 min; $5,8-\mathrm{diHOD})$, peak $2 *\left(10.9 \mathrm{~min}, \lambda_{\max } 218 \mathrm{~nm}\right.$; lactonized $5,8-$ diHOD), and peak 3 (I5.I min; 8-HOD), the major fatty acid metabolites. RP-HPLC analysis and purification of the fatty acid products were carried out on a Cosmosil 5C I8-AR (5 $\mu \mathrm{m}$; $250 \times 4.6 \mathrm{~mm}$ i.d.; Nacalai Tesque, Kyoto, Japan) reversedphase column using a gradient system (solvent A: methanol/ water/acetic acid (50:50:0.01, v/v/v); solvent B: methanol/ water/acetic acid $(95: 5: 0.0 \mathrm{I}, \mathrm{v} / \mathrm{v} / \mathrm{v}))$ with the following gradient program: $45 \%$ solvent $A$ for I min, followed by a linear increase of solvent B up to $100 \%$ within 10 min and finally an isocratic post-run at $100 \%$ solvent $B$ for $10 \mathrm{~min}$. The flow-rate was I $\mathrm{mL} / \mathrm{min}$. Reference compounds of dihydroxy fatty acids had a retention time of 9-II min, whereas monohydroxy fatty acid references eluted between $15-18 \mathrm{~min}$.

similar that of the TMS ether of methyl 8,11-dihydroxy octadecanoate [7]. The GC retention time and mass spectrum of the non-hydrogenated sample and the GC retention time and mass spectrum of TMS ether of methyl 8,11dihydroxy-9,12-octadecadienoate showed that the major fatty acid product in RP-HPLC peak 1 (Fig. 1) was 8,11dihydroxy octadecadienoic acid (8,11-diHOD) [7].

Hydrogenated RP-HPLC peak 2 (Fig. 1) as TMS ether of methyl ester derivative was separated on GC and one dominant peak was present in the chromatogram. The mass spectrum showed characteristic peaks stemming from cleavages around the two oxygenated $\mathrm{C}$ atoms that indicated the presence of TMS ether of methyl 5,8-dihydroxy octadecanoate. Comparison of the mass spectrum from hydrogenated and non-hydrogenated samples showed that the TMS ether of methyl 5,8-dihydroxy octadecanoate was derived from the TMS ether of methyl 5,8-dihydroxy-9,12-octadecadienoate. This was evidenced by the molecular ion at $m / z 470$ and by the characteristic fragments resulting from cleavage around the double bonds and oxygenated $\mathrm{C}$ atoms [8]. Thus RPHPLC peak 2 (Fig. 1) proved to be 5,8-diHOD.

RP-HPLC peak $2 *$ was analyzed as a part of RP-HPLC peak 2 , due to overlap. Hydrogenation of the TMS ether deriv- 
ative showed peaks stemming from cleavage around an oxygenated C-atom. The molecular ion at $\mathrm{m} / \mathrm{z} 370$ evidenced that this compound was TMS ether of lactonized 5,8-dihydroxyoctadecanoate. Comparing the hydrogenated sample with the non-hydrogenated sample showed that TMS ether of lactonized 5,8-dihydroxy octadecanoate probably originated from lactonized 5,8-diHOD.

\section{GCIMS analysis of monohydroxy fatty acids (RP-HPLC peak 3)}

In the GC chromatogram of the hydrogenated monohydroxy fatty acids of RP-HPLC peak 3 (Fig. 1) as TMS ethers of methyl ester derivatives, one prominent peak was present. The mass spectrum identified it as a mixture of the TMS ethers of methyl 8-hydroxy octadecanoate, methyl 10-hydroxy octadecanoate and a small amount of methyl 9-hydroxy octadecanoate. Also, a small peak of methyl 13-hydroxy octadecanoate was present in the GC chromatogram. In the GC/MS analysis of the corresponding non-hydrogenated monohydroxy fatty acids as TMS ethers of methyl ester derivatives, three peaks were visible in the GC chromatogram. Reference compounds indicated that GC peak 1 (18.3 min) was TMS ether of methyl 8-hydroxy octadecadienoate because of the fragmentation pattern and retention time of the non-hydrogenated sample [7]. The mass spectrum of TMS ether of methyl 10hydroxy octadecanoate, GC peak 2 (18.4 min), showed that this compound originated from 10-hydroxy octadecadienoic acid (10-HOD). The mass spectrum of GC peak 4 (19.1 $\mathrm{min})$ and the mass spectra of reference compounds showed that TMS ethers of methyl 13-hydroxy octadecanoate and methyl 9-hydroxy decanoate were derived from 13-hydroxy octadecadienoic acid (13-HOD) and 9-hydroxy octadecadienoic acid (9-HOD), respectively. Thus, RP-HPLC peak 3 (Fig. 1) was composed of 8 HOD (20), 10-HOD (18), 13-HOD (1) and 9-HOD (1).

GC/MS analysis of monohydroxy fatty acids eluting after RP-HPLC peak 3 (Fig. 1) as TMS ethers of methyl ester derivatives showed that a small amount of 8-HOM was also present (data not shown).

\section{Characteristics of oxylipin formation}

Incubation with [U-13 $\mathrm{C}] 18: 2$ showed that all oxygenated fatty acid products (RP-HPLC peak 1 to peak 3, Fig. 1) represented a mixture of converted 18:2 from endogenous and exogenous sources. The conversion of $500 \mathrm{nmol}$ exogenously supplied 18:2 was about $50 \%$ of the total conversion, as judged by the ratio of $\left[\mathrm{U}-{ }^{13} \mathrm{C}\right]$ labeled fragments to unlabeled fragments on GC/MS. In a reaction with 20:4 was shown that 20:4 it was not converted by an $A$. niger N402 crude extract.

\section{Endogenous oxylipins}

Endogenous oxylipins of A. niger N402 biomass were extracted and analyzed on GC/MS. Oxylipin levels were very low when compared to the total ion-current of the internal standard 17:0. Traces of 5,8-diHOD, 8,11diHOD, 8-HOD, 10-HOD, 13-HOD and 8-HOM were detected, however, oxylipin levels were generally just above background. Similar results were obtained for $A$. niger UU-A049.1, A. niger $\triangle p p o A$ (UU-A050.3), A. niger $\triangle p p o D$ (UU-A051.26) and A.nidulans WG096.

\section{Identification of three putative A. niger dioxygenase genes, ppoA, ppoC and ppoD}

A search of the A. niger N402 genomic database identified three putative dioxygenase genes $p p o A, p p o C$ and $p p o D$ that are located on chromosomes 6,4 and 3, respectively, and contained 6, 12, and 11 introns, respectively. The deduced amino acid sequences of PpoA (1080 aa, $120 \mathrm{kD})$, PpoC (1110 aa, $125 \mathrm{kD}$ ) and PpoD (1164 aa, $131 \mathrm{kD}$ ) represented proteins with strong homology to G. graminis LDS. A. niger PpoA and PpoC were closely related to A. nidulans PpoA and PpoC (Table 1). Comparing the sequence of $A$. niger PpoD with those of PpoA, PpoB and PpoC from $A$. nidulans showed that $A$. niger ppoD had strongest similarity to A. nidulans PpoA and PpoC and not to A. nidulans PpoB (Table 1).

In analogy with G. graminis LDS and A. nidulans Ppo's, A. niger PpoA, PpoC and PpoD showed homology to animal PGS $\left(E\right.$-values $>7 \times 10^{-21} ;>3 \times 10^{-24} ;>3 \times 10^{-18}$, respectively). A. niger PpoA, PpoC and PpoD also contained the distal $(202 ; 246 ; 265$, respectively) and proximal (377; $424 ; 444$, respectively) His, and Tyr $(374 ; 420 ; 441$, respectively) residues, essential for catalytic activity of PGS. Amino acid analysis of the predicted proximal His domain revealed that $\mathrm{PpoD}$ differed from the other Aspergillus Ppo's in having a Phe (443) instead of a Trp residue between the proximal His and Tyr residues and that a Lys, conserved in the other Ppo's, was replaced by a Gln (453) residue (Fig. 2)

Putative A. niger PpoA and PpoC contained the proline knot motif that targets proteins to oil bodies in plants $[4,9]$. In contrast $A$. niger PpoD did not contain the proline knot motif, the third Pro residue was replaced by an Arg residue (Fig. 3).

\section{Phenotypic characterization of A. niger transformants}

To study the connection of the A. niger putative dioxygenase genes to oxylipin formation and reproduction, $p p o A$ and $p p o D$ were inactivated by homologous recombination of the domain encoding the catalytic site with the $\arg B$ cassette. A. niger $\triangle p p o A$ and $\triangle p p o D$ mutants had no alterations in radial growth. Also their response to osmotic, oxidative and temperature stress, and combinations thereof, did not differ from the reaction of the wild type. No effect on sporulation was observed for the $p p o A$ and $p p o D$ disruption strains or the $p p o A$ multicopy strain. However, a $34 \%$ reduction in conidiospores was observed in the $p p o C$ 
Table I: Comparisson of predicted A. niger putative dioxygenases PpoA, PpoC and PpoD

\begin{tabular}{|c|c|c|c|c|c|}
\hline Protein & Protein & $E$-value & $\begin{array}{c}\text { Identities } \\
\%\end{array}$ & $\begin{array}{c}\text { Positives } \\
\%\end{array}$ & $\begin{array}{c}\text { Gaps } \\
\%\end{array}$ \\
\hline \multirow[t]{4}{*}{ A. niger $\mathrm{PpoA}$} & A. nidulans PpoA & 0 & 69 & 81 & 7 \\
\hline & A. nidulans $\mathrm{PpoC}$ & 0 & 37 & 56 & 10 \\
\hline & A. nidulans $\mathrm{PpoB}$ & $1 \times 10^{-68}$ & 43 & 53 & 21 \\
\hline & G. graminis LDS & 0 & 45 & 60 & 8 \\
\hline \multirow[t]{4}{*}{ A. niger PpoC } & A. nidulans $\mathrm{PpoC}$ & 0 & 60 & 75 & 10 \\
\hline & A. nidulans $\mathrm{PpoA}$ & 0 & 47 & 64 & 10 \\
\hline & A. nidulans $\mathrm{PpoB}$ & $8 \times 10^{-86}$ & 39 & 51 & 20 \\
\hline & G. graminis LDS & $3 \times 10^{-174}$ & 41 & 58 & 10 \\
\hline \multirow[t]{4}{*}{ A. niger PpoD } & A. nidulans $\mathrm{PpoA}$ & $5 \times 10^{-177}$ & 38 & 55 & 11 \\
\hline & A. nidulans $\mathrm{PpoC}$ & $8 \times 10^{-161}$ & 31 & 46 & 12 \\
\hline & A. nidulans $\mathrm{PpoB}$ & $5 \times 10-70$ & 41 & 52 & 19 \\
\hline & G. graminis LDS & $1 \times 10^{-143}$ & 38 & 55 & 2 \\
\hline
\end{tabular}

multicopy strain. In experiments where linoleic acid was added, all strains showed reduced conidiospore counts compared to the wild type.

\section{A. niger microarray analysis}

Analysis of expression levels of $A$. niger putative dioxygenases $p p o A, p p o C$ and $p p o D$ showed that the three genes were expressed from the center to the periphery of the $A$. niger colonies grown on maltose, however, the level of expression differed (Fig. 4). The genes $p p o A$ and $p p o D$ were expressed mainly in the periphery, while levels of $p p o C$ expression were equally distributed throughout the colony and low in comparison to the expression of $p p o A$ and $p p o D$. Similar results were obtained during growth on Dxylose (data not shown). Since the A. niger strains were grown in sandwiched cultures, the formation of conidia was suppressed. Expression profiles of dioxygenase genes may be different in sporulating colonies.

\section{Discussion}

The goal of this study was to investigate whether or not oxylipins and dioxygenase genes, that are related to both asexual and sexual reproduction, are present in the asexual fungus $A$. niger.

Using RP-HPLC and GC/MS, this study demonstrated that A. niger converted 18:2 mainly into 8,11-diHOD, 5,8diHOD, lactonized 5,8-diHOD, 8-HOD and 10-HOD. The reaction with [U-13C] $18: 2$ showed that these compounds were produced from a mixture of exogenously added and endogenously present 18:2.

The presence of 5,8-diHOD, lactonized 5,8-diHOD, 8HOD and 10-HOD, but not the presence of 8,11-diHOD were reported in A. nidulans $[8,10-13]$ and related to sexual reproduction [2-4]. Interestingly, in the present study 8,11-diHOD was one of the oxylipins formed by $A$. nidu-

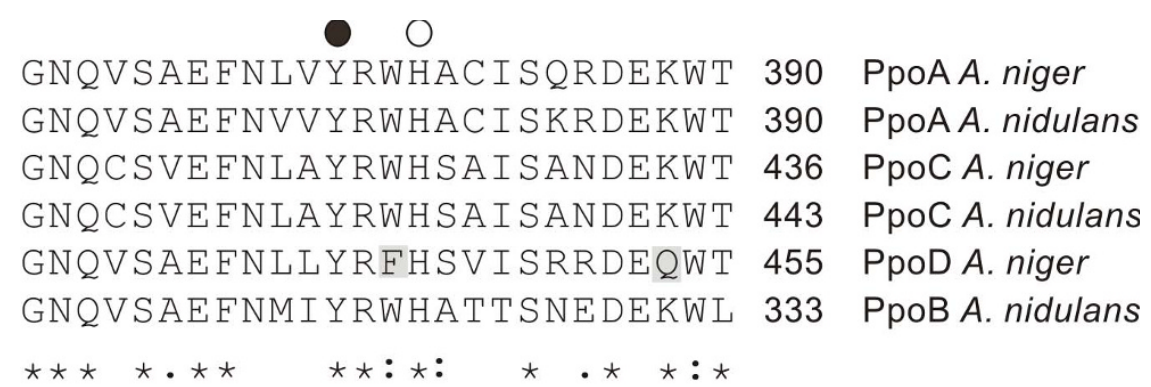

Figure 2

Amino acid alignment of the predicted proximal His domain in A. niger PpoA, PpoC and PpoD to A. nidulans PpoA, PpoB and PpoC. Identical amino acids are marked with asterisks; similar amino acids are marked with colons. The proximal His and the Tyr residue important for catalysis in PGS are marked with $\bigcirc$ and $\bigcirc$ respectively. Deviating amino acids (Phe instead of Trp, and Gln instead of Lys), in A. niger PpoD are indicated in grey. 


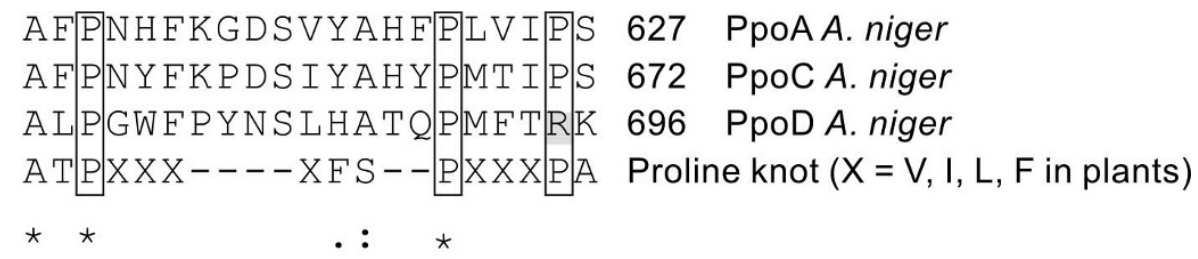

Figure 3

Amino acid alignment of the predicted proline knot motif in A. niger PpoA, PpoC and PpoD to the proline knot motif in plants $[9,24]$. Identical amino acids are marked with asterisks; similar amino acids are marked with colons. The conserved Pro residues are indicated with boxes. The third Pro residue in A. niger PpoD is replaced with an Arg residue, indicated in grey.

lans. During the preparation of this manuscript, a study was published showing that the asexual fungus A. fumigatus also produced 5,8-diHOD, 8,11-diHOD 8-HOD and 10-HOD [13]. This indicates that A. niger, A. nidulans and A. fumigatus all produce the same oxylipins.

Analysis of the A. niger genome revealed that this fungus contains three putative dioxygenase genes, $p p o A, p p o C$ and $p p o D$. A $p p o B$ homologue was not present. A. niger transformants lacking the $p p o A$ or $p p o D$ gene were not altered in their ability to produce oxylipins and sporulation. A reduction in conidiospore formation was observed in the $p p o C$ multicopy strain. In contrast, in A. nidulans ppoA, $p p o B$ or $p p o C$ were found to be connected to oxylipin pro-

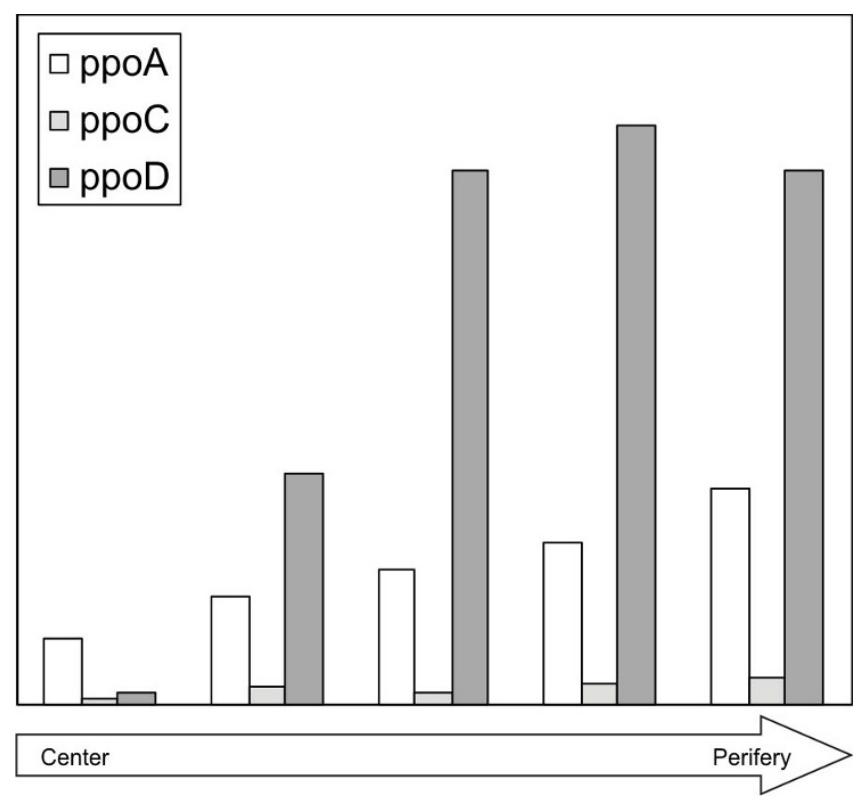

Figure 4

Microarray analysis of expression levels of $A$. niger putative dioxygenase genes $p p \circ A, p p \circ C$ and $p p o D$ on maltose. Five distinct zones were taken from the center to the perifery. Indicated are the relative expression levels. duction and to modification of sexual and asexual sporulation. Deletion of $p p o A, p p o B$ or $p p o C$ was demonstrated to reduce the level of $8-\mathrm{HOD}, 8-\mathrm{HOM}$ and $8-\mathrm{HOM}$, respectively [2-4]. But a later study showed that deletion of $p p o A$ led to a reduction of 8-HOD and 5,8-diHOD formation and that elimination of $p p o C$ reduced 10-HOD formation [13]. The removal of $p p o B$ did not alter oxylipin production [13]. In addition, deletion of $p p o A$ or $p p o B$ from the A. nidulans genome increased the ratio of asexual to sexual spores $[3,4]$. Elimination of $p p o C$ on the other hand, significantly reduced the ratio of asexual to sexual spores [2]. Absence of a phenotype for the disruption strains of $A$. niger for $p p o A$ and $p p o D$, could suggest that they are non-essential or that they in fact have the same function. Future studies into these genes should include construction of double-disruptants. The inability to isolate $p p o C$ disruptants might suggest that this is an essential gene in A. niger even though this is not the case in A. nidulans [2] and could possibly indicate significant differences in the role of these genes in different fungi. When linoleic acid was added, all strains showed reduced asexual sporulation compared to the wild type, suggesting that addition of linoleic acid could not be compensated for when the production of the different Ppo's is altered in $A$. niger.

A. niger PpoD had deviating amino acid residues in the vicinity of the proximal His domain and did not contain the proline knot motif (Fig. 3). This motif targets plant proteins to oil bodies and it has been demonstrated that fungi target such proteins to oil bodies as well [14]. In addition, the proline knot is predicted to facilitate the formation of an antiparallel $\alpha$-helix or $\beta$-strand [9]. Therefore, A. niger PpoD likely differs from the other Ppo's in its three dimensional structure It could be argued that the presence of $p p o D$ instead of $p p o B$ in $A$. niger is related to the reproductive differences between $A$. niger and A. nidulans. However, this seems unlikely since the genomes of Aspergillus clavatus, Aspergillus oryzae and Aspergillus terreus, all not known to reproduce sexually, do contain $p p o B$ homologues. Additionally, Histoplasma capsulatum, a fun- 
gus belonging to the same class as the Aspergilli, contains $p p o D$ and also does not contain $p p o B$, but is able to produce sexual spores [3]. Expression analysis of $A$. niger $p p o A, p p o C$ and $p p o D$ shows that these genes are expressed and their expression levels depend on the fungus' developmental stage (Fig. 4). It should be noted that A. niger is heterothallic and requires mating between two isolates with different mating types. Despite the fact that $A$. niger appears to contain all the genes required for a sexual cycle, until now, no sexual cycle has been observed for $A$. niger on any of a broad range of growth conditions (Paul Dyer, personal communication). In contrast, A. nidulans is a homothallic species in which both mating types are present in a single strain and can therefore cross with itself. This difference might hint towards different strategies for regulation of sexual and asexual development. Studies of these genes in other homothallic and heterothallic Aspergilli, could demonstrate whether this is a general difference between homothallic and heterothallic species. This could include the presence or absence of expression of specific dioxygenase genes.

Strictly asexual species are considered an evolutionary endpoint, and truly asexual species are thought to be extremely rare [5]. Sequencing of fungal genomes and comparative analysis of sexual and asexual species show that fungi that have long been considered asexual organisms, may have a latent potential for sexual reproduction $[1,6]$. Nevertheless, the presence of sex related genes alone, does not confirm sexual reproduction.

\section{Conclusion}

This study shows that $A$. niger produces the same oxylipins and has similar dioxygenase genes as A. nidulans. Even though, the functionality of these genes remains as yet to be proven, their presence could point towards the existence of sexual reproduction in A. niger or a broader role for the gene products in physiology, than just sexual development.

\section{Methods \\ Materials}

All chemicals used were commercially obtained and of analytical grade. Linoleic acid (9Z,12Z-octadecadienoic acid, 18:2, 99\% pure), arachidonic acid, 5Z,8Z,11Z,14Z- eicosatetraenoic acid, 20:4, 99\% pure) and margaric acid (heptadecanoic acid, 17:0, 99\% pure) were obtained from Sigma (St. Louis, MO). [U- $\left.{ }^{13} \mathrm{C}\right]$ 18:2 (99\% pure) was obtained from Isotec (Matheson Trigas, Irving, TX). Solutions of $30 \mathrm{mM}$ fatty acid were stored in methanol under $\mathrm{N}_{2}$ at $-20^{\circ} \mathrm{C}$ until use.

\section{Strains, media and culture conditions}

Aspergillus strains used are listed in Table 2. Cultures were grown in minimal medium containing trace elements and $1 \%$ glucose as carbon source, unless otherwise indicated in the text [15]. Appropriate supplements ( $8 \mu \mathrm{M}$ nicotinamide, $1.5 \mathrm{mM}$ leucine, $5 \mathrm{mM}$ uridine) were added to the media to complement auxotrophic mutations. Aspergillus cultures were inoculated with $10^{6}$ spores $/ \mathrm{ml}$ and grown at $30^{\circ} \mathrm{C}$ on a rotary shaker (Inova 2300; New Brunswick Scientific, Edison, NJ) at $250 \mathrm{rpm}$. For growth on solid media $1.5 \%$ of agar was added. Strains were grown in $25 \mathrm{ml}$ of liquid medium in Petri dishes under stationary conditions at $30^{\circ} \mathrm{C}$. Alternatively, strains were grown in $50 \mathrm{ml}$ of liquid medium at $30^{\circ} \mathrm{C}$ in a rotary shaker at $250 \mathrm{rpm}$. Mycelial mats were collected after $72 \mathrm{~h}$, dried between filter paper sheets and frozen in liquid nitrogen.

\section{Oxylipin characterization and analysis of enzymatic capacity}

For analysis of endogenously present oxylipins, samples were lyophilized, weighed and homogenized mechanically using a microdismembrator (B. Braun GmbH, Melsungen, Germany). Free fatty acids and their derivatives were extracted with $80 \%$ methanol $1: 10(\mathrm{w} / \mathrm{v})$, centrifuged at $4^{\circ} \mathrm{C}, 2500 \times \mathrm{g}$ for $20 \mathrm{~min}$ and recovered by solid phase extraction (SPE, Oasis HLB 200 mg; Waters, Milford, MA). 17:0 was used as an internal standard.

The enzymatic capacity to oxygenate fatty acids of Aspergillus strains was examined as follows. Samples were homogenized, extracted with phosphate buffer ( $50 \mathrm{mM}$ sodium phosphate $\mathrm{pH} 6.5,5: 1 \mathrm{w} / \mathrm{v}$ ) and centrifuged at $4^{\circ} \mathrm{C}, 2500$ $\times \mathrm{g}$ for $20 \mathrm{~min}$. The supernatant (crude extract) was filtered through cheesecloth and used immediately. Typically, $4 \mathrm{~mL}$ phosphate buffer was mixed with $1 \mathrm{~mL}$ crude extract, rigorously stirred and incubated with $120 \mu \mathrm{M}$ substrate for 30-45 min at room temperature under a contin-

Table 2: Aspergillus strains used in this study

\begin{tabular}{|c|c|}
\hline Strain & Genotype \\
\hline A. niger N402 (FGSCA733) & $\operatorname{cspAl}$ \\
\hline A. niger UU-A049.I & nicAI, leuAI, pyrA6, $\triangle \arg B:: A$. niger $\arg B$ \\
\hline A. niger $\triangle p p o A$ UU-A050.3 & nicAI, leuAI, pyrA6, $\triangle \operatorname{argB}::$ ppoA disruption construct \\
\hline A. niger $\triangle p p o D \cup U-A 051.26$ & nicAI, leuAI, pyrA6, $\triangle \operatorname{argB}::$ ppoD disruption construct \\
\hline A. nidulans WG096 (FGSCI87) & pabaAI, yA2 \\
\hline
\end{tabular}


uous flow of $\mathrm{O}_{2}$. Fatty acids and reaction products were recovered directly by $\mathrm{SPE}$.

\section{RP-HPLC and GCIMS analysis}

SPE eluates were concentrated under $\mathrm{N}_{2}$, and analyzed by RP-HPLC. Analysis by GC/MS of the fatty acid products as TMS ethers of methyl ester derivatives was performed as described previously [16]. The fatty acid methylation reagent was diazomethane. For GC/MS analysis, samples were analyzed before and after hydrogenation. Oxylipins were identified by mass spectrum on the basis of their fragmentation patterns.

\section{Nucleic acid manipulations}

The amino acid sequence of Gaeumannomyces graminis linoleate diol synthase (LDS) [17] was used to perform a BLASTp search of the A. niger N402 [18] genomic database (DSM food specialties, Delft, The Netherlands). Three putative dioxygenase genes ( $p p \circ A$; GeneID: 4990997, ppoC; GeneID: 4985482 and ppoD; GeneID: 4979282) were identified that predicted proteins with high similarity to LDS. These genes were aligned to the ppo genes from A. nidulans and to the LDS from G. graminis and a phylogenetic tree was created using the ClustalW program http://www.ebi.ac.uk/clustalw. Pairwise scores between amino acids were represented as the number of identities and positives.
Primers to amplify fragments for complete gene (constructs containing promoter, gene and terminator) and disruption constructs were based upon the A. niger N402 genome sequence. These primers introduced restriction sites at either site of the amplified fragment during a PCR reaction (Table 3 ). A. niger genomic DNA was isolated using previously described techniques and used as the PCR template [19]. PCRs were carried out with AccuTaq LA $^{\mathrm{TM}}$ DNA polymerase according to the manufacturer's protocol (Sigma) and the annealing temperature varied between $52^{\circ} \mathrm{C}$ and $60^{\circ} \mathrm{C}$. Amplified PCR products were cloned into the pGEMTeasy vector (Promega, Madison, WI) and used to transform competent Escherichia coli DH5 $\alpha$. Positive clones containing the fragments for complete gene or disruption constructs were analyzed by restriction mapping and sequence comparisons to the NCBI genetic database using the tBLASTn algorithm http:/ /www.ncbi.nlm.nih.gov.

\section{Creation of disruption and complete gene constructs}

Primers for fragments for disruption constructs were designed at the 5' and 3' flanking regions of predicted catalytic domains of PpoA, PpoC and PpoD. These catalytic domains were identified by ClustalW alignment of predicted PpoA, PpoC and PpoD to the LDS from G. graminis of which the catalytic domain has been identified [17]. Amino acids 202 to 883 for PpoA and aminoacids 224 to

Table 3: Primers used in this study

Sequence $5^{\prime} \rightarrow 3^{\prime}$

\begin{tabular}{|c|c|}
\hline Constructs of comple & \\
\hline PMWOI2 & \\
\hline ppoA-dw & GAGGTGGGTCTTGTTTG \\
\hline ppoA-up & GACAAACAGGGAGTTGC \\
\hline pMW036 & \\
\hline ppoD-dw & GATTTCTTCCAGCTGGC \\
\hline ppoD-up & GCTACAGCTACAGCTAC \\
\hline Disruption construct & \\
\hline pMW05I & \\
\hline ppoA3'-Nsil-dw & ATGCATGGTGGCAAACCAAGCC \\
\hline ppoA3'-Kpnl-up & GGTACCGGTGAGGAGCACTACTTG \\
\hline ppoA5'-HindlII-dw & AAGCTTATTTGTAGAGTCGAGG \\
\hline ppoA5'-Sphl-up & GCATGCCATGCTTACCGTGAATG \\
\hline pMW06I & \\
\hline ppoD5'-Kpnl-dw & GGTACCTTCCAGCTGGCATTGGTG \\
\hline ppoD5'-BamHI-up & GGATCCGTGCAGGGCCTTGAGCC \\
\hline ppoD3'-Sphl-dw & GCATGCTGAAGCGCAACGTCTAAC \\
\hline ppoD3'-HindIII-up & AAGCTTCAGCCCGTAGTTCTG \\
\hline
\end{tabular}


1010 for PpoD were deleted. These contained for both PpoA and PpoD the distal (202; 265, respectively) and proximal $(377 ; 444$, respectively) His, and Tyr $(374 ; 441$, respectively) residues, essential for catalytic activity of PGS. Primers for complete genes were designed approximately $80 \mathrm{bp}$ outside of the coding region.

Disruption constructs for $p p o A, p p o C$ and $p p o D$, including the $\arg B$ marker gene, were created as follows [20]. First, the $5^{\prime}$ and $3^{\prime}$ flanking regions were amplified by PCR introducing the indicated restriction sites (Table 3 ). The amplified products were digested from pGEMTeasy, separated on $0.8 \%$ agarose gel and isolated. The flanks were ligated into the pUC19 vector (Fermentas, Ontario, Canada) containing the $\arg B$ cassette (pRV542) previously digested with the appropriate restriction enzymes resulting in the disruption constructs for $p p o A, p p o C$ and $p p o D$. Disruption constructs were linearized by digestion with KpnI/HindIII and used for A. niger transformations.

\section{A. niger transformations}

Protoplasts were prepared from A. niger UU-A049.1 as described and transformed using polyethylene glycol [21]. Transformation of A. niger UU-A049.1 with ppoA and $p p o D$ disruption constructs created transformants to ArginineB prototrophy with the catalytic domain of the corresponding gene product deleted. Three independent Aspergillus niger transformations did not result in the isolation of a $p p o C$ disruptant and we were therefore not able to analyze this gene disruption. Transformants were purified by repeated streaking of conidia. Gene replacement and ectopic integration of the $\arg B$ marker gene were checked by PCR and Southern analysis using internal fragments as probes.

\section{Probe construction and Southern analysis}

Constructs of complete genes of $p p o A$ and $p p o D$ were digested with EcoRV and SphI, respectively, yielding internal probes for the encoding region of the catalytic domain. Fragments were separated on an $0.8 \%$ agarose gel, isolated and randomly labeled with $\left[\alpha{ }^{-32} \mathrm{P}\right] \mathrm{dCTP}$. This resulted in 1082 and 1146 bp fragments for $p p o A$ and a 1241 bp fragment for $p p o D$. Chromosomal DNA of $A$. niger transformants was digested with the appropriate restriction enzymes. Hybridization with radioactive probes was done as described, except that washing of the filters was done at $65^{\circ} \mathrm{C}$ [22]. Positive transformants, lacking the signals from the internal probes on the Southernblot, were selected and used for further characterization.

\section{Phenotypic characterization of A. niger transformants}

Characterization of $A$. niger transformants was performed on solid minimal medium containing $1 \%$ glucose and supplemented with or without $1 \mathrm{M} \mathrm{NaCl}$ and/or $0.01 \%$ $\mathrm{H}_{2} \mathrm{O}_{2}$ at $30^{\circ} \mathrm{C}$ and $42^{\circ} \mathrm{C}$. Spots of $10000,1000,100$ and
10 conidia were pipetted on each plate and incubated. Strains A. niger 49.1 and A. niger N402 were used as wild type. Spore production studies were carried out on plates containing $25 \mathrm{~mL}$ solid minimal medium and $1 \%$ glucose [3]. For each plate a $5 \mathrm{~mL}$ top layer of cool melted $0.6 \%$ agar minimal medium and $1 \%$ glucose containing $10^{7}$ conidia of the appropriate strain was added. In some cases $1.5 \%$ methanol or $1.5 \%$ methanol and $10 \mu \mathrm{g} / \mathrm{mL}$ linoleic acid were added to both agar layers. Cultures were incubated at $30^{\circ} \mathrm{C}$. Cores of $16 \mathrm{~mm}$ diameter were removed from each plates and homogenized for $1 \mathrm{~min}$ in $3 \mathrm{~mL}$ sterile water supplemented with $0.01 \%$ Tween- 80 to facilitate release of the hydrophobic conidia. Spores were counted by using a haemacytometer.

\section{A. niger microarray analysis}

A. niger $\mathrm{N} 402$ was grown at $30^{\circ} \mathrm{C}$ as sandwiched cultures [23] in minimal medium [15] with $25 \mathrm{mM}$ maltose or 25 $\mathrm{mM}$ D-xylose as carbon source. Zonal mycelial samples from 3 sandwich cultures were combined and used for RNA analysis. Mycelium was ground using a microdismembrator and RNA was extracted using TRIzol reagent (Invitrogen, Carlsbad, CA) according to the instructions of the manufacturer. RNA was purified using Nucleospin RNA clean up (Macherey-Nagel GmbH, Düren, Germany). Concentration of RNA was measured at $\lambda_{260}$. Quality of the RNA was analyzed on an Agilent 2100 BioAnalyzer using the RNA6000 labchip kit (Agilent Technologies, Palo Alto, CA). Biotin-labeled antisense cRNA was generated by labeling 20 or $2 \mu \mathrm{g}$ of total RNA with the BioArray High Yield RNA transcription labeling kit (ENZO) or the Affymetrix Eukaryotic One-Cycle Target Labeling and Control Reagent package, respectively. The quality of the cRNA was checked using the Agilent 2100 bioanalyzer. The labeled cRNA was hybridized to Affymetrix A. niger Genechips. Absolute values of expression were calculated from the scanned array using the Affymetrix GCOS software after an automated process of washing and staining. Microarray Suite Affymetrix v5.1 (Affymatrix Inc., Santa Clara, CA), Spotfire DecisionSite (Spotfire Inc, Somerville, MA), GeneData Expressionist Analyst V Pro 2.0.18 (GeneData, Basel, Switzerland) and the R statistical environment http://www.r-cran.org were used for data analyses.

\section{Abbreviations}

17:0: margaric acid, heptadecanoic acid; 18:2: linoleic acid, 9Z,12Z-octadecadienoic acid; 20:4: arachidonic acid, 5Z,8Z,11Z,14Z-eicosatetraenoic acid; 8-HOD: 8hydroxy octadecadienoic acid; 8-HOM: 8-hydroxy octadecamonoenoic acid; 9-HOD: 9-hydroxy octadecadienoic acid; 13-HOD: 13-hydroxy octadecadienoic acid; 10HOD: 10-hydroxy octadecadienoic acid; 8,11-diHOD: 8,11-dihydroxy octadecadienoic acid; 5,8-diHOD: 5,8dihydroxy octadecadienoic acid; PGS: prostaglandin syn- 
thase; LDS: linoleate diol synthase; SPE: solid phase extraction; TMS: trimethylsilyl.

\section{Authors' contributions}

This work was performed as part of the PhD thesis for MWW. MWW carried out most experimentation. SICK performed the spore production studies. All experiments were supervised by RPV. All authors have contributed to the experimental and analytical design. MWW, RPV, JFGV (thesis advisor) and GAV (thesis advisor) wrote the manuscript. All authors have read and approved the final manuscript.

\section{Acknowledgements}

The authors would like to thank Dr A.M. Levin and prof. Dr H.A.B. Wösten for providing the data of the $A$. niger microarray analysis.

\section{References}

I. Pel HJ, de Winde JH, Archer DB, Dyer PS, Hofmann G, Schaap PJ, Turner G, de Vries RP, Albang R, Albermann K, et al:: Genome sequencing and analysis of the versatile cell factory Aspergillus niger CBS 5 I 3.88. Nature biotechnology 2007, 25:22 I-23I.

2. Tsitsigiannis DI, Kowieski TM, Zarnowski R, Keller NP: Endogenous lipogenic regulators of spore balance in Aspergillus nidulans. Eukaryot Cell 2004, 3:1398-14II.

3. Tsitsigiannis DI, Kowieski TM, Zarnowski R, Keller NP: Three putative oxylipin biosynthetic genes integrate sexual and asexual development in Aspergillus nidulans. Microbiol 2005, | 5 |:|809-|82|.

4. Tsitsigiannis DI, Zarnowski R, Keller NP: The lipid body protein, PpoA, coordinates sexual and asexual sporulation in Aspergillus nidulans. J Biol Chem 2004, 279: I I 344- I I 353.

5. Taylor JW, Jacobson DJ, Fisher MC: The evolution of asexua fungi: reproduction, speciation and classification. Ann Rev Phytopathol 1999, 37: 197-246.

6. Dyer PS, Paoletti M: Reproduction in Aspergillus fumigatus: sexuality in a supposedly asexual species? Med Mycol 2005 43:S7-SI4.

7. Wadman MW, van Zadelhoff G, Hamberg M, Visser T, Veldink GA Vliegenthart JFG: Conversion of linoleic acid into novel oxylipins by the mushroom Agaricus bisporus. Lipids 2005, 40:1163-1170

8. Calvo AM, Gardner HW, Keller NP. Genetic connection between fatty acid metabolism and sporulation in Aspergillus nidulans. J Biol Chem 200I, 276:25766-25774.

9. Abell BM, Holbrook LA, Abenes M, Murphy DJ, Hills MJ, Moloney MM: Role of the proline knot motif in oleosin endoplasmic reticulum topology and oil body targeting. Plant Cell 1997. 9:| $48|-| 493$

10. Champe SP, EI-Zayat AAE: Isolation of a sexual sporulation hormone from Aspergillus nidulans. J Bacteriol 1989, I7 I:3982-3988.

II. Mazur P, Meyers HV, Nakanishi K, El-Zayat AAE, Champe SP: Structural elucidation of sporogenic fatty acid metabolites from Aspergillus nidulans. Tetrahedron Lett 1990, 31:3837-3840.

12. Mazur P, Nakanishi K, El-Zayat AAE, Champe SP: Structure and synthesis of sporogenic psi factors from Aspergillus nidulans. Chem Soc Chemm Comm 1991:|486-1487.

13. Garscha U, Jerneren F, Chung D, Keller NP, Hamberg M, Oliw EH: Identification of dioxygenases required for aspergillus development: Studies of products, stereochemistry and the reaction mechanism. J Biol Chem 2007, 282:34707-347I8.

14. Ting JTL, Balsamo RA, Ratnayake C, Huang AHC: Oleosin of plant seed oil bodies is correctly targeted to the lipid bodies in transformed yeast. J Biol Chem 1997, 272:3699-3706

15. de Vries RP, Burgers K, Vondervoort PJl van de, Frisvad JC, Samson RA, Visser J: A new black Aspergillus species, A. vadensis, is a promising host for homologous and heterologous protein production. Appl Environ Microbiol 2004, 70:3954-3959.
16. van Zadelhoff G, Veldink GA, Vliegenthart JFG: With anandamide as substrate plant 5-lipoxygenases behave like I I-lipoxygenases. Biochem Biophys Res Commun 1998, 248:33-38.

17. Hornsten L, Su C, Osbourn AE, Garosi P, Hellman U, Wernstedt C, Oliw EH: Cloning of linoleate diol synthase reveals homology with prostaglandin H synthases. I Biol Chem 1999, 274:28219-28224.

18. Bos CJ, Debets AJM, Swart K, Huybers A, Kobus G, M SS: Genetic analysis and the construction of master strains for assignment of genes to six linkage groups in Aspergillus niger. Curr Genet 1988, 14:437-443.

19. de Graaff LH, Broek HW] van den, Visser J: Isolation and transformation of the pyruvate kinase gene of Aspergillus nidulans. Curr Genet 1988, 13:315-321.

20. Lenouvel F, Vondervoort PJI van de, Visser J: Disruption of the Aspergillus niger argB gene: a tool for transformation. Curr Genet 2002, $41: 425-432$.

21. Kusters-van Someren MA, Harmsen JA, Kester HC, Visser J: Structure of the Aspergillus niger pelA gene and itsexpression in Aspergillus niger and Aspergillus nidulans. Curr Genet 1991, 20:293-299.

22. de Vries RP, Vondervoort PJI van de, Hendriks L, Belt M van de, Visser J: Regulation of the alpha-glucuronidase-encoding gene (aguA) from Aspergillus niger. Mol Genet Genomics 2002, 268:96-102.

23. Wösten HAB, Mouhka SM, McLaughlin PMJ, Sietsma JH, Wessels $\mathrm{JGH}$ : Localization of growth and excretion of proteins in Aspergillus niger. J Gen Microbiol I991, 137:2017-2023.

24. Murphy $D J$ : The biogenesis and functions of lipid bodies in animals, plants and microorganisms. Progress in lipid research 200I, 40:325-438

Publish with BioMed Central and every scientist can read your work free of charge

"BioMed Central will be the most significant development for disseminating the results of biomedical research in our lifetime. "

Sir Paul Nurse, Cancer Research UK

Your research papers will be:

- available free of charge to the entire biomedical community

- peer reviewed and published immediately upon acceptance

- cited in PubMed and archived on PubMed Central

- yours - you keep the copyright 